\title{
La felicidad, un asunto de niños
}

\section{Happiness, a children's subject}

\author{
Mabel Goretty Chala Trujillo' \\ Universidad Nacional Abierta y a Distancia, UNAD
}

\begin{abstract}
Resumen
La felicidad es un estado anhelado por los seres humanos y a la vez, es un tema que resulta de interés para los investigadores. Este artículo describe las experiencias de felicidad descritas por 60 niños y niñas, matriculados en instituciones públicas de Bogotá, Neiva y Pasto (Colombia) durante el año 2013. Desde el enfoque cualitativo y el diseño metodológico Relatos de Vida, se buscó conocer el significado sobre felicidad que construyen los las niñas y los niños escolarizados con edades en edades entre 9 y 11 años en las mencionadas ciudades. Los participantes se refieren a la felicidad como una vivencia de la cotidianidad, disfrutando de compañía de seres queridos, del juego y de actividades sencillas en las que pueden compartir con otros. Se sienten felices cuando sus necesidades básicas están satisfechas, cuando son reconocidos y aceptados y/o cuando pueden interactuar con sus pares o sus familiares positivamente. La discusión se centra en la concordancia entre la vivencia de emociones positivas, lo cual genera estados de bienestar y satisfacción y el significativo aporte que pueden brindar los adultos a la felicidad de niños y niñas, cuando los reconocen como seres singulares, con necesidades particulares, y se ocupan de satisfacerlas.
\end{abstract}

Palabras clave: felicidad, emociones, reconocimiento, identidad, vínculos afectivos.

\begin{abstract}
Happiness is a coveted status in humans yet, is a topic of interest to researchers. This article describes the experiences of happiness described by 60 children enrolled in public institutions in Bogotá, Neiva and Pasto during 2013. From the qualitative approach and the methodological design of Life Stories, one sought to know the meaning of happiness that both boys and girls aged between 9 and 11 years old and enrolled in school, have in the afore mentioned cities. Participants refer to happiness as an experience of daily life, enjoying the company of loved ones, playing and enjoying simple activities where they can share with others. They are happy when their basic needs are met, when they are recognized and accepted and/ or when they can interact positively with their peers or their family. The discussion focuses on the correlation between the experience of positive emotions which generates states of wellness and satisfaction and the significant contribution that adults can make to children's happiness when they recognize children as unique beings, with particular needs and they try to meet those needs.
\end{abstract}

Keywords: happiness, emotions, recognition, identity, emotional ties.

Recibido: 31 de julio de 2015

Aceptado: 10 de septiembre de 2015

1 Mabel Goretty Chala Trujillo. Psicóloga, Especialista en Gerencia de Salud Ocupacional, Magister en Educación Superior. Coinvestigadora en el Grupo de Investigación "Psicología, Desarrollo Emocional y Educación”. Universidad Nacional Abierta y a Distancia. Correo: mabel.chala@ unad.edu.co 


\section{Introducción}

¿Alguna vez ha observado cómo un niño pelea con su amigo y sólo pasan escasos minutos para verlos nuevamente jugando juntos?, ¿recuerda alguna escena en la que un adulto reprende a un niño y, casi inmediatamente, el niño interactúa con el adulto como si nada hubiera pasado?, ¿ha pensado que los niños vivencian una "natural alegría de vivir"? Respuestas a estas preguntas se han encontrado en el estudio realizado en 2013, por el grupo de investigación Psicología Desarrollo Emocional y Educación de la UNAD, sobre las experiencias que pueden definir la felicidad para niños y niñas escolarizados. El objetivo general del estudio fue "Comprender los significados que sobre felicidad construyen los niños y las niñas escolarizados(as) en edades entre 9 y 11 años en las ciudades de Bogotá, Neiva y Pasto".

Eninterpretación, seformulandosconcordancias: la primera, la relación entre la vivencia natural de las emociones y la felicidad, en donde los niños y las niñas expresan que se sienten felices cuando "dialogo con mi mamá, juego con mis amigos, celebramos los cumpleaños o visitamos a la abuela", esto genera emociones positivas que se perciben como "estados transitorios que dan felicidad" y la segunda, la relación entre la valoración y el reconocimiento propiciado por los adultos genera sensaciones de bienestar y satisfacción, asociados a la felicidad. Los niños y las niñas comentan que se sienten felices "cuando comparto mi papá, cuando la profesora me trata bien, cuando me regalan cosas o cuando me dan mi comida favorita". Estas dos ideas básicas, se desarrollan a lo largo del texto.

La felicidad ha sido un "estado" deseado por los seres humanos a lo largo de la historia. Ya Aristóteles definió la felicidad como "actividad del alma" asociada a la "virtud":

"La felicidad no es algo dado por la naturaleza sino es el resultado del obrar... del hombre, es decir, requiere un esfuerzo. De ahí que el concepto finalista de felicidad lleva por sí mismo al concepto de la virtud como una actitud de esfuerzo hacia la vida buena" (Estermann, 2001).
Platón y Arístipo (Siglo IV a.C.), así como Epicuro y Aristóteles (Siglo III a.C.) describieron la "Felicidad" como el propósito central de la experiencia humana. Como tema de disertación desde la Psicología, el estudio de la felicidad es reciente (finales del siglo XX). Desde la ciencia, se ha intentado definirla y medirla, lo que es una tarea compleja, particularmente porque desde la psicología el término tiene varias connotaciones. Para medirla hay dos tendencias generales entre la comunidad científica, la primera apunta a la felicidad colectiva y la segunda a la felicidad individual. En este artículo, se hace referencia a esta última, desde la cual se estudian experiencias y percepciones vitales en términos del nivel de satisfacción pasajera o duradera en diferentes ámbitos de la vida y en la percepción de la vida como un todo (felicidad individual), evaluándose desde dos fuentes de información que aporta el sujeto: los afectos (aspecto emocional o afectivo) y los pensamientos (aspecto cognitivo). Esto, en razón a que el ser humano es capaz de experimentar, sentir o vivenciar satisfacciones o sufrimientos y valorar cognitivamente estas percepciones, así como comparar su realidad con el ideal que tiene en mente, entonces, la felicidad es algo que el hombre tiene en la mente y por lo tanto se puede medir haciendo preguntas, es decir pidiendo a la gente en qué medida disfruta de la vida como un todo (Veenhoven, 2009).

\section{Metodología}

Desde el Enfoque Cualitativo, con el diseño metodológico Relatos de Vida se analizaron los discursos acerca del significado que han construido sobre el ser feliz, de 60 niños y niñas en edad escolar, distribuidos proporcionalmente en las ciudades de Bogotá, Neiva y Pasto. La conformación de cada uno de los grupos se hizo de manera intencional teniendo en cuenta criterios inclusión como estar matriculado en la Institución, contar con los debidos consentimientos informados, que los participantes estuvieran en edades entre los 9 y los 11 años, y que estuvieran interesados en participar. 
Mediante entrevistas y grupos focales, se conocieron las experiencias autorreferenciales, es decir, cómo los niños y las niñas conciben la felicidad y la expresan; los escenarios y las prácticas, esto es, dónde los participantes se sienten felices, qué cosas o actividades los hacen felices, con quién o quiénes se sienten felices; y las explicaciones sobre la felicidad, relacionadas con las condiciones que los niños y las niñas consideran permiten que alguien sea feliz; cómo los niños y las niñas explican las condiciones que deben cumplirse para que alguien sea feliz.

\section{Hallazgos}

Con base en las categorías mencionadas, se encontró que los niños y las niñas de instituciones educativas públicas de las ciudades de Bogotá, Neiva y Pasto, se refieren a la felicidad como un concepto abstracto, una vivencia inexpresable, que se percibe como bienestar, como emoción que genera reacciones fisiológicas, que se manifiesta en gestos como la sonrisa y en posturas corporales como la disposición para la acción; que forja pensamientos y acciones positivas $y$ que se exterioriza en metáforas como "siento un corrientazo", "es como una chispita". Esto se puede evidenciar en el siguiente testimonio: "Me pasa un corrientazo en todo el cuerpo, el corazón me empieza a latir duro, duro, duro y ahí siento la felicidad" (Niño de 10 años).

Una de las cuestiones que mayor felicidad genera a los niños y las niñas es el compartir con otros, interactuar positivamente y mantener relaciones interpersonales adecuadas, es decir, la felicidad se centra en relaciones interpersonales positivas en donde la interacción auténtica produce bienestar. A su vez, la felicidad se expresa en acciones concretas que facilitan el contacto interpersonal y el compartir. Para los niños y las niñas, es muy importante el tipo y la calidad de los vínculos que establecen en sus relaciones interpersonales, esto es fuente de bienestar y felicidad. Los siguientes testimonios, dan cuenta de lo expresado: "Poder compartir muchísimo tiempo con mis padres, familia" (Niño de 10 años). "Me hace feliz tener buenas

\section{FELICIDAD SE VIVENCIA COMO}

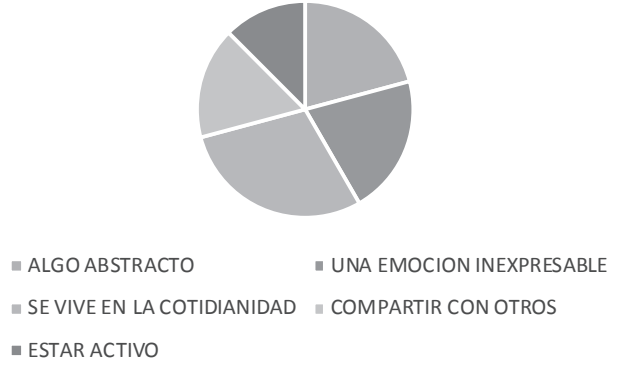

Figura 1. Cómo se vivencia la felicidad.

DIMENSIONES QUE HACEN FELIZ A UN NIÑO O NIÑA PARTICIPANTE EN EL ESTUDIO

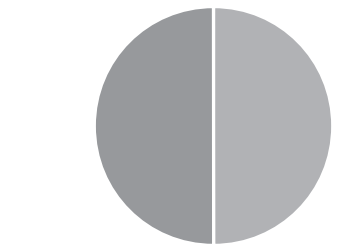

- VIVENCIA NATURAL DE LAS EMOCIONES

- VALORACIÓN Y RECONOCIMIENTO DE LA PERSONA

Figura 2. Dimensiones que hacen feliz a un niño o niña.

FELICIDAD EN NIÑOS Y ADULTOS

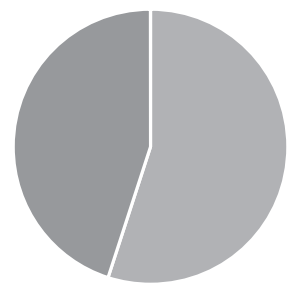

= Niños y niñas: Felicidad Particular a los sucesos vitales del individuo n Adultos: Felicidad Global

Figura 3. Felicidad en niños y adultos.

relaciones, que me relacione bien con mis compañeros de clase. No meterme en peleas porque uno es el que sale emproblemado" (Niño de 11 años).

Se es feliz en la cotidianidad, cuando se hacen cosas propias de los niños, como jugar, bailar, cantar; el juego y las actividades con otros son fuente de bienestar y felicidad. Por otra parte, se siente felicidad cuando se es reconocido y se comparte con otros, la felicidad implica disposición para compartir con otros. "Los video 
juegos, la música, el baile, el fútbol, montar en los carros chocones, jugar con los amigos" (Niño de 9 años). "Cuando me invitan a jugar o cuando vamos a pasear" (Niña de 10 años).

En otros casos, la felicidad de un niño depende del estado de los padres, así, los adultos y los niños y las niñas se influyen mutuamente para dar y sentir felicidad. De la misma manera, los niños y las niñas participantes en el estudio consideran que su buen comportamiento genera felicidad en adultos, así como el amor y la estabilidad familiar: "Cuando papá llega de trabajar y habla conmigo o cuando mi mamá me habla, me pregunta qué me pasa..." (Niña de 9 años); "No me siento feliz cuando mi mamá me contesta mal" (Niña de 10 años); "Cuando saco buenas notas y mis papás me felicitan y también mis tíos y tías y nos vamos al río, piscina o a comer helado" (Niña de 11 años).

Como puede verse, para los niños y las niñas participantes, se es feliz cuando se disfruta de cosas sencillas en la vida cotidiana, se comparte con otros y se reconoce la dignidad humana. Ser feliz es estar activo y posibilitar la interacción con otros... como lo propuso Aristóteles, "felicidad es igual a acción", acción genuina, tranquila, sencilla... "Ser feliz" es entonces una "cosa de niños", dado que un niño puede expresar la vivencia de una genuina felicidad y disfrutar momentos plenos en situaciones simples. "Soy feliz cuando estoy en mi casa, en el estudio, cuando estoy paseando y cuando me sacan al centro, a los parques" (Niña de 10 años). "Me hace feliz estar con mi familia, con mis amigos y con mis compañeros de clase" (Niña de 11 años).

\section{Discusión}

Con lo anterior, se plantea entonces que niños y niñas son felices básicamente por dos circunstancias: La primera, al vivenciar las emociones de manera natural, esto es, de manera intensa y pasajera. La segunda, cuando son reconocidos por sus cuidadores, familiares y/o adultos cercanos. Cuando un niño o una niña no ha sido abrumado por experiencias difíciles o traumáticas que le alejen de sus expresiones naturales ni que le dificulten el ser reconocido de manera genuina por los otros, cercanos a él, puede experimentar muchos momentos de felicidad. Que un niño o niña pueda comportarse de manera natural significa que puede pensar y expresarse sin ser reprimido, puede sentir y emocionarse sin ser castigado o invalidado. Esto implica la posibilidad de establecer relaciones interpersonales apropiadas que se constituyen en generadoras de bienestar.

Este tipo de relaciones se empiezan a tejer desde el nacimiento, cuando inicia el diálogo entre la madre y el niño y ambos logran lo que Schaffer, (1977) citado por Craig, (1992) denomina reciprocidad o sincronía entre lactante y cuidador. A partir de allí se configura el primer vínculo que se constituye en el prototipo con el que el niño se relacionará más adelante. De este modo, llorar manifestando una necesidad que es debidamente atendida por el cuidador, genera en el bebé emociones placenteras que se van interiorizando para dar paso a un desarrollo socioafectivo pleno y satisfactorio. En este sentido se empieza a experimentar de manera segura la posibilidad de expresar una necesidad que es satisfecha. Garelli y Montouri, (1997) anotan que las características de los vínculos afectivos establecidos en la infancia permiten comprender por qué algunos niños crecen felices y seguros de sí mismos, otros ansiosos y deprimidos, y otros agresivos e incluso antisociales.

Cuando se establecen los primeros vínculos de manera segura, el niño o la niña continúa su desarrollo con la posibilidad de experimentar sus emociones de manera original es, decir, sintiéndolas y "dejándolas ir", como corresponde a la naturaleza humana. La emoción, se entiende como un impulso arraigado que conlleva a la acción $y$, en todos los casos, genera una reacción fisiológica transitoria. Es por esto que un niño después de pelear con un amigo o de ser reprendido, nuevamente interactúa como si nada hubiera sucedido, porque vivencia la emoción, la "suelta" y retoma su presente.

Así, se esclarece la manera en que los niños y las niñas dan significado a la felicidad: como un conjunto de momentos felices o una serie de experiencias transitorias en las que vivencian 
emociones positivas. En este sentido, Diener (2000), citado por Caycho (2010), plantea que existe una Felicidad Particular a los sucesos vitales del individuo y una Felicidad Global que equivale a "la percepción subjetiva de bienestar y satisfacción". Esta última correspondería a la felicidad esperada por los adultos, mientras que la primera, sería la percepción que corresponde a los niños y las niñas, según lo que han expresado.

Diener, (2000) citado por Caycho (2010), indica que la felicidad corresponde a la evaluación subjetiva que cada cual hace de su propia vida y que puede ser contemplada desde su totalidad o desde alguna de sus facetas. Los niños y las niñas la contemplan como diferentes momentos que se vivencian plena y auténticamente y entonces la felicidad es "como una chispita", "cuando me dan besos y abrazos", "cuando me palpita el corazón porque me dan un regalo" o "cuando mi mamá está feliz porque he sacado buenas notas".

La segunda condición que se ha mencionado como requisito para la felicidad de un niño o una niña es el sentirse reconocido. "Reconocer" es explorar algo o alguien de manera cuidadosa, revisar algo para conocer lo que contiene. Para el tema que se está tratando, se diría que reconocer a un niño o niña es ocuparse de saber quién es, identificarlo en primer lugar como ser humano íntegro y completo. Reconocer a un niño o niña es, entonces, legitimar su condición humana, conocer sus pensamientos, emociones, actitudes, características de personalidad, motivaciones, necesidades y por ende, validarlas y/o satisfacerlas. Esto implicaría garantizar la satisfacción de sus necesidades actuando conforme el niño o la niña van cambiando en sus fases de desarrollo; diferenciarlo de los demás y valorarlo por lo que es, porque cuando se reconoce, se concreta la individualización y se aporta al desarrollo de la identidad personal. “... la identidad no es una mera representación del tipo cognitivo; es, sobre todo, un "sentimiento de identidad": sentirse una persona, alguien entre los demás..." (Craig, 1992). Cuando el niño se "siente alguien", cuando se siente valorado, identificado por sí mismo y por los otros, se siente feliz.
En contraste, la negligencia y el rechazo de los adultos que forman parte de su micromundo genera consecuencias desastrosas en la formación del carácter y los estados de ánimo del niño. Según Rhoner (1986), citado por Gracia y cols., (2005) se considera rechazo parental, la ausencia o privación significativa de calor, afecto o amor de los padres hacia sus hijos. Los niños pueden percibir rechazo de forma hostil y agresiva, negligente, o indiferenciada; indistintamente si el rechazo proviene del padre o de la madre, el clima familiar de rechazo marca el desajuste social y psicológico del niño, lo que influirá posteriormente, cuando el adolescente intente lograr un sentido de identidad (Erikson, 1965 citado por Craig, 1992).

Con lo anterior, ser feliz es entonces, un "asunto de niños", porque ser feliz es conservar la alegría de vivir, ser auténticos, desarrollar el potencial, apreciar las cosas sencillas de la vida, reconocer lo valioso en sí mismos y en los demás. El niño y la niña podrán conservar su "natural alegría de vivir" en la medida en que sus cuidadores o adultos responsables de ellos, propicien un clima positivo, les permitan expresarse genuinamente $y$ les faciliten el desarrollo en el cual puedan promover vínculos seguros que potencien un individuo diferenciado, singular, auténtico, capaz de disfrutar aspectos pasajeros de la vida que le den "satisfacciones instantáneas" que, a su vez, favorezcan la construcción del camino hacia su "satisfacción vital o felicidad" (Veenhoven, 2009).

\section{Referencias}

Bowlby, J. (1998). El apego y la pérdida. La separación. Vol. 2. Ed. España: Paidós Iberoamérica.

Carter, C. (2011). El aprendizaje de la felicidad. España: Urano.

Caycho Rodríguez, T. (2010). Psicología de la felicidad. Introducción a la psicología positiva. Psicología. 28.2 (June 2010): 411(4). Academic OneFile. Gale. Univ.

Estermann, J. (2001). Historia de la Filosofía. Quito: Abya Yala.

Craig, G. J. (1992). Desarrollo Psicológico. México: Prentice Hall. 
Garelli, J. y Montouri, E. (1997). Vínculo afectivo materno-filial en la primera infancia y teoría del attachment. Revista práctica pediátrica. Vol. 95. pp 192-195. Archivo argentino de pediatría. Buenos Aires, Argentina.

González-Quiñones, J. C.; Restrepo-Chavarriaga, G. (2010). Prevalencia de felicidad en ciclos vitales y relación con redes de apoyo en población colombiana. Revista de Salud Pública, vol. 12, núm. 2, abril, 2010, pp. 228-238. Universidad Nacional de Colombia. Bogotá, Colombia. Redalyc. Sistema de Información Científica. Red de Revistas Científicas de América Latina, el Caribe, España y Portugal.

Gracia, E., Lila, M. y Musitu G. (2005). Rechazo parental y ajuste psicológico y social de los hijos. Revista Salud Mental. Vol. $28 \mathrm{~N}^{\circ}$ 2. pp 73-81. Instituto mexicano de psiquiatría Ramón de la Fuente. Distrito federal. México.

Haidt, J. (2006). La hipótesis de la felicidad. Barcelona: Gedisa. p. 120 citado por De Zubiría Samper, Miguel. ¿Cómo ser feliz? Las conclusiones de recientes investigaciones científicas psicológicas. Módulo 1. Bogotá: Liga Contra el Suicidio.

Lyubomirsky, S. (2008). La ciencia de la felicidad. Crecimiento y Salud. Barcelona: Ediciones Urano.

Moyano Díaz, E. y Alvarado, N. (2007). Bienestar subjetivo: midiendo satisfacción vital, felicidad y salud en población chilena de la Región Maule. Chile: Universidad de Talca. Revista Universum No 22 Vol. 2.

Nettle, D. Felicidad. (2005). La ciencia tras la sonrisa. España: Ares y Mares.

Perinat, A. (2002). Desarrollo socioafectivo en niños de 2 a 12 años. Barcelona: UOC. Vallejo-Nájera, A. (2012). Hablemos de felicidad. España: Ediciones Urano.

Veenhoven, R. (2009). Medidas de la Felicidad Nacional Bruta. Psychosocial Intervention - Intervención Psicosocial, Diciembre - Sin mes, 279-299. Vol. 18 [citado 2012-08-15]. Disponible en Internet: http://www.redalyc.org/src/inicio/ArtPdfRed. jsp?iCve=179814227007. ISSN 1132-0559. 